\title{
The Calcium Isotope Composition of the Lower Mantle and bulk Earth
}

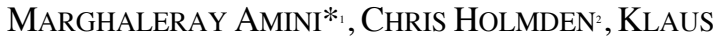 \\ PETER JOCHUM ${ }^{3}$
}

PCIGR, Earth, Ocean \& Atmospheric Sciences, University of British Columbia, Vancouver BC, V6T-1Z4, Canada

${ }^{2}$ SIL, Department of Geological Sciences, University of Saskatchewan, Saskatoon SK, Canada

${ }_{3}^{3}$ Max Planck Institute for Chemistry, Mainz, Germany

(*correspondence: mamini@eoas.ubc.ca)

The relevance of the Earth's $\mathrm{Ca}$ element and isotope inventory became increasingly evident with the emergence of global climate simulations linking the $\mathrm{Ca}$ cycle to the global $\mathrm{CO}_{2}$ system [e.g. 1,2]. The determination of the bulk Earth $\mathrm{Ca}$ isotope value is challenged by $\mathrm{Ca}$ isotope variations in igneous rocks and between different meteorite groups [3, 4]. Recent studies have constrained the bulk silicate Earth $\mathrm{Ca}$ isotope composition by empirical and/or theoretical approaches [e.g. 5, 6]. To date, a $\delta^{4440} \mathrm{Ca}_{\text {(SRMYI5a) }}$ of $\sim 1.05 \%$ is estimated for the bulk silicate Earth based on model calculations considering inter-mineral fractionation between orthopyroxene and clinopyroxene as the predominant $\mathrm{Ca}$-bearing phases in the mantle [5]. This value implies an enstatite/ordinary chondritic Earth for $\mathrm{Ca}$ isotopes while being significantly higher than carbonaceous (C-)chondrites [6]. However, if deducing the $\mathrm{Ca}$ isotope bulk silicate Earth value is based on equilibrium $\mathrm{Ca}$ isotope fractionation at high temperatures [7], then subsequently the $\mathrm{Ca}$ isotope composition of the lower mantle reservoir containing Ca-(silicate-)perovskite needs to be explored and/or a $\mathrm{Ca}$ isotope fractionation between upper and lower mantle precluded in order to extrapolate to bulk Earth.

Here, we discuss several scenarious for the Ca isotope bulk Earth composition including nominally lower values for the lower mantle and hence implying a C-chondritic Earth. We base our discussion on a compilation of recent and previous data for $\mathrm{Ca}$ isotopes in various terrestrial and extraterrestrial materials and on theoretical calculations for equilibrium $\mathrm{Ca}$ isotope fractionation. Ultimately, we attempt to further constrain the $\mathrm{Ca}$ isotope composition and evolution of the Earth and potentially also for other planetary bodies.

[1] Milliman (1993) GBC 7(4), 927-957; [2] Griffith et al. (2008) Science 322, 2060-2062; [3] Skulan et al. (1997) GCA 12, 1671; [4] Simon and Depaolo (2010) EPSL 289, 457-466; [5] Huang et al. (2010) EPSL 292, 337-344; [6] Valdes et al. (2014) EPSL 394, 135-145; [7] Feng et al. (2014) GCA 143, 132-142. 